Delft University of Technology

\title{
Sparse Signal Bands Selection for Precise Time-based Ranging in Terrestrial Positioning
}

Dun, H.; Tiberius, Chistian C.J.M.; Diouf, Cherif; Janssen, Gerard J.M.

DOI

10.1109/PLANS46316.2020.9110197

Publication date

2020

Document Version

Accepted author manuscript

Published in

2020 IEEE/ION Position, Location and Navigation Symposium (PLANS)

\section{Citation (APA)}

Dun, H., Tiberius, C. C. J. M., Diouf, C., \& Janssen, G. J. M. (2020). Sparse Signal Bands Selection for Precise Time-based Ranging in Terrestrial Positioning. In 2020 IEEE/ION Position, Location and Navigation Symposium (PLANS) (pp. 1372-1380). [9110197] IEEE .

https://doi.org/10.1109/PLANS46316.2020.9110197

Important note

To cite this publication, please use the final published version (if applicable).

Please check the document version above.

\section{Copyright}

Other than for strictly personal use, it is not permitted to download, forward or distribute the text or part of it, without the consent of the author(s) and/or copyright holder(s), unless the work is under an open content license such as Creative Commons.

Takedown policy

Please contact us and provide details if you believe this document breaches copyrights.

We will remove access to the work immediately and investigate your claim. 


\section{Sparse Signal Bands Selection for Precise Time-based Ranging in Terrestrial Positioning}

\author{
Han Dun, Christian C. J. M. Tiberius, Cherif Diouf \\ Geoscience and Remote Sensing \\ Delft University of Technology \\ Delft, The Netherlands
}

\author{
Gerard J. M. Janssen \\ Circuits and Systems \\ Delft University of Technology \\ Delft, The Netherlands
}

\begin{abstract}
Time-based ranging accuracy is inversely proportional to the signal bandwidth. A larger the signal bandwidth leads to a higher accuracy of time delay estimation, but more complex hardware is needed. Alternatively, we explore the idea of using multiple narrow signal bands (e.g., $10 \mathrm{MHz}$ of each) to create a large virtual signal bandwidth, which maintains the spectral efficiency but largely improves the ranging accuracy. Considering the impact of multipath, the propagation delay of the LoS path is computed from the estimated channel impulse response (CIR). In this paper, we propose an approach to sparsely select signal bands for ranging and positioning based on convex optimization. The Cramér-Rao lower bound (CRLB) for the propagation delay and gain estimators, as a performance criterion, is employed in the constraint of the optimization. The CRLB is derived in a two-path channel, so that the accuracy and the correlation between the LoS path and the reflection are taken into account. Experiments are conducted in a laboratory environment to illustrate the proposed signal design methodology dedicated for ranging with a sub-decimeter accuracy.

Index Terms-time delay estimation, ranging, OFDM, convex optimization, Cramér-Rao lower bound, multipath channel
\end{abstract}

\section{INTRODUCTION}

In a ranging system for positioning and navigation, the propagation time delay is the most straightforward measurement to retrieve geometric information. The accuracy of delay estimation is inversely proportional to the signal bandwidth. In GNSS, because of its relatively narrow signal bandwidth, it becomes difficult to distinguish the direct line-of-sight (LoS) path from close-in reflections. Thus, multipath is a significant error source in high precision GNSS applications [1]. Given a fixed and relatively narrow signal bandwidth, the maximization of the Gabor bandwidth is proven to be effective in providing robustness against multipath [2]. Alternatively, in a terrestrial positioning system, we can potentially occupy a large signal bandwidth for time-based ranging. For example, the signals transmitted in the ISM bands (e.g., 2400-2500, 5875-5925 $\mathrm{MHz}$ ), ultra-wide band (UWB) signals, and other opportunistic signals that can be modified for ranging and positioning.

However, using a very large signal bandwidth for ranging requires an analog-to-digital converter (ADC) with a very high sample rate, and is also not efficient in terms of spectrum usage. In addition, to estimate the propagation delay from a large amount of samples also requires substantial processing power.

This research is supported by the Netherlands Organization for Scientific Research (NWO) through the project SuperGPS under Grant 13970.
Instead of occupying the entire available signal bandwidth, in [3] we explored the idea of using multiple signal bands that are sparsely located within the available signal spectrum, to create a large virtual signal bandwidth, which has been referred to as a sparse multiband signal. As a follow-up, in this paper, we focus on the signal design, specifically on how to sparsely select multiple signal bands from the available signal spectrum, such that we occupy little total spectrum bandwidth, and can still achieve a favorable ranging accuracy.

In a time-based ranging system, we are most interested in the propagation delay of the direct LoS path, which can in principle be derived from the channel impulse response (CIR). To obtain the CIR, the propagation delay and the gain should be estimated for each path. Although we only use time delay estimates for ranging, the estimates of the gain are also useful in a multipath channel as they may be indicative for a direct LoS path, a blocked path, or a reflection. Thus, we derive the Cramér-Rao lower bound (CRLB) for the propagation delay and gain estimators in a two-path channel, as a performance criterion to design the signal. The CRLB derived for a two path channel considers the covariance of the estimators between the LoS path and a reflection [4]. That is why we need to consider a channel with at least two paths. When the estimators of the LoS path are highly correlated with the estimators of a reflection, we may not be able to distinguish the LoS component from the reflection. Consequently, the LoS time delay estimate can be largely affected by multipath.

In this paper, the baseband signal in each signal band, as an example, is modulated in orthogonal frequency division multiplexing (OFDM). Then, we aim to select optimal and possibly sparse OFDM signal bands for ranging and positioning, which can meet the desired ranging accuracy and with a capability to distinguish certain reflections.

The rest of the paper is organized as follows. Section II presents the CRLB of the propagation delay and gain in a two path channel. A convex optimization problem is formulated for signal bands selection in section III. Then, section IV presents a sparsity-prompt time delay estimation method. Simulation and experimental results are shown in section V. Lastly, the conclusion is drawn in section VI.

Notation: $\mathbb{E}\{\cdot\}$ denotes the expectation operation. $\mathbb{R}(\mathbb{C})$ is used to indicate a real (complex) variable. (.) denotes a random 
variable. $(\cdot)$ denotes an estimate. $(\cdot)^{\mathrm{T}},(\cdot)^{*}$ and $(\cdot)^{\mathrm{H}}$ denote transposition, conjugate and Hermitian operation, respectively. Upper boldface letters (e.g., $\boldsymbol{F}$ ) are used for matrices, and $[\cdot]_{i j}$ denotes the element in the $i$-th row and the $j$-th column of a matrix. Lower boldface letters (e.g., $\boldsymbol{r}$ ) are used for column vectors. A normal lower letter or $[\cdot]_{i}$ is used to denote an element in a column vector. $\mathcal{I}_{N}$ is the identity matrix of size $N . \mathbf{1}_{N}$ denotes a $N \times 1$ vector of one. $\Re\{\cdot\}$ and $\Im\{\cdot\}$ denote the real part and imaginary part of a complex value. $\|x\|_{2}=$ $\left(\boldsymbol{x}^{\mathrm{T}} \boldsymbol{x}\right)^{1 / 2}$ denotes the Euclidean norm (i.e., $l_{2}$ norm). $\|\boldsymbol{x}\|_{1}=$ $\left|x_{1}\right|+\ldots+\left|x_{2}\right|$ denotes the sum-absolute-value (i.e., $l_{1}$ norm).

\section{Cramér-Rao lower Bound for Two Path Channel}

Assuming that the received OFDM signal, which contains $N$ subcarriers, is only perturbed by complex Gaussian noise. Thus, there are $N$ samples taken from one OFDM symbol. The distribution of the observation is defined as follows,

$$
\underline{\boldsymbol{r}}[\boldsymbol{\theta}] \sim \mathcal{C N}\left(\boldsymbol{\mu}_{r}(\boldsymbol{\theta}), \boldsymbol{C}_{r}(\boldsymbol{\theta})\right), \quad \boldsymbol{C}_{r}(\boldsymbol{\theta})=\boldsymbol{C}_{r}=\sigma^{2} \mathcal{I}_{N},
$$

where $\boldsymbol{\theta}$ denotes unknown parameters, $\boldsymbol{\mu}_{r}(\boldsymbol{\theta})=\mathbb{E}(\underline{\boldsymbol{r}}[\boldsymbol{\theta}])$, and $\boldsymbol{C}_{r}(\boldsymbol{\theta})$ denotes the variance matrix of the observations.

In practice, the signal is always received in a multipath channel. For the purpose of ranging and positioning, although we are only interested in the propagation delay of the LoS path, the gain of each path is also useful to help to select the LoS path in a multipath channel. Therefore, we estimate the delay and the gain not only for the LoS path, but also for the reflection and the estimators can be assumed to be unbiased in an actual multipath channel. The CRLB of any unbiased estimator is given by [5]

$$
C R L B(\hat{\boldsymbol{\theta}})=\boldsymbol{F}^{-1}(\boldsymbol{\theta}),
$$

where $\boldsymbol{F}(\boldsymbol{\theta})$ denotes the Fisher information matrix (FIM), given by

$$
\begin{aligned}
{[\boldsymbol{F}(\boldsymbol{\theta})]_{i j}=} & \operatorname{tr}\left[\boldsymbol{C}_{\boldsymbol{r}}^{-1}(\boldsymbol{\theta}) \frac{\partial \boldsymbol{C}_{\boldsymbol{r}}(\boldsymbol{\theta})}{\partial \theta_{i}} \boldsymbol{C}_{\boldsymbol{r}}^{-1}(\boldsymbol{\theta}) \frac{\partial \boldsymbol{C}_{\boldsymbol{r}}(\boldsymbol{\theta})}{\partial \theta_{j}}\right] \\
& +2 \Re\left[\frac{\partial \boldsymbol{\mu}_{\boldsymbol{r}}^{\mathrm{H}}(\boldsymbol{\theta})}{\partial \theta_{i}} \boldsymbol{C}_{r}^{-1}(\boldsymbol{\theta}) \frac{\partial \boldsymbol{\mu}_{\boldsymbol{r}}(\boldsymbol{\theta})}{\partial \theta_{j}}\right],
\end{aligned}
$$

For simplicity in the following derivation, we consider a two path channel as an example, but this can be expanded to multiple reflections. Hence, the set of unknown parameters is defined by $\boldsymbol{\theta}$,

$$
\boldsymbol{\theta}=\left[\begin{array}{llll}
\tau_{1} & \tau_{2} & \alpha_{1} & \alpha_{2}
\end{array}\right]^{\mathrm{T}}=\left[\begin{array}{l}
\boldsymbol{\tau} \\
\boldsymbol{\alpha}
\end{array}\right]
$$

where $\tau_{1}, \alpha_{1}$ and $\tau_{2}, \alpha_{2}$ denote the delay and gain of the $\operatorname{LoS}$ path and the reflection, respectively.

Now, we consider that $M$ OFDM signal bands are available for time delay estimation, as shown in Fig.1.

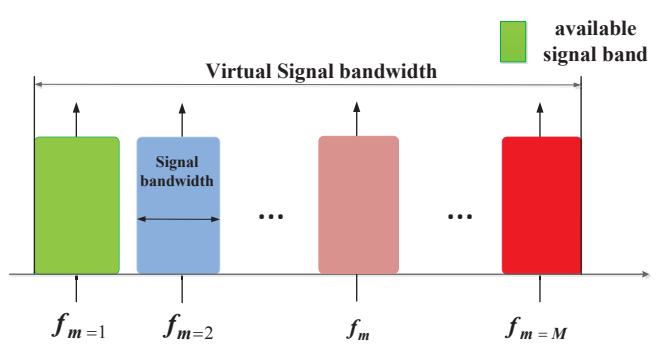

Fig. 1. The spectrum of the multiband OFDM signal for a time-based ranging system, consisting of $M$ bands.

First, we derive the CRLB based on a single band OFDM signal $\underline{r}_{m}[n ; \boldsymbol{\theta}]$ (e.g., obtained from the $m$-th band), which is written by

$$
\begin{aligned}
& \underline{r}_{m}[n ; \boldsymbol{\theta}]= \frac{\alpha_{1}}{\sqrt{N}} \sum_{i=-N / 2}^{N / 2-1} c_{i} \exp \left(j 2 \pi \frac{i}{N} n\right) \\
& \cdot \exp \left(-j 2 \pi\left(f_{m}+f_{i}\right) \tau_{1}\right) \\
&+\frac{\alpha_{2}}{\sqrt{N}} \sum_{i=-N / 2}^{N / 2-1} c_{i} \exp \left(j 2 \pi \frac{i}{N} n\right) \\
& \cdot \exp \left(-j 2 \pi\left(f_{m}+f_{i}\right) \tau_{2}\right)+\underline{e}_{m}[n] \\
& n=0,1, \ldots, N-1 ; m=1,2, \ldots, M,
\end{aligned}
$$

where $n$ denotes the sample index, $i$ and $N$ respectively denote the index of the subcarrier and the total number of subcarriers in each band, $c_{i}$ denotes the data modulated on the $i$-th subcarrier, $f_{m}$ denotes the central carrier frequency of the $m$-th band (see Fig.1). In addition, $M$ denotes the total number of signal bands available in the signal spectrum, $\underline{e}$ denotes the complex additive white Gaussian noise (AWGN).

Usually, the carrier phase is not taken into consideration when we estimate the propagation delay from a received baseband signal. However, given multiple signal bands that are modulated on different carrier frequencies, the received baseband signals from the different bands contain different phase rotations by the same propagation delay. In order to benefit from its virtual signal bandwidth, we should consider these phase rotations, so that we can maintain their frequency relation across multiple signal bands. Thus, the virtual signal bandwidth is not determined by the actual carrier frequency, but by the difference between the carrier frequencies. The carrier frequency $f_{m}$ in the local replica for the $m$-th band is offset in the sequel, for instance, by the smallest central carrier frequency (i.e., $f_{m=1}$ )

$$
\tilde{f}_{m}=f_{m}-f_{m=1}, \quad m=1,2, \ldots, M .
$$

The derivation of the FIM in a two-path channel can be found in appendix A. To simplify the notation of the FIM, 
here we first define

$$
\boldsymbol{f}_{m}=\left[\begin{array}{c}
f_{i=-N / 2} \\
f_{i=-N / 2+1} \\
\vdots \\
f_{i=N / 2-1}
\end{array}\right]+\tilde{f}_{m}, \quad \boldsymbol{f}_{m}^{2}=\boldsymbol{f}_{m} \odot \boldsymbol{f}_{m}
$$

where the vector $\boldsymbol{f}_{m}$ contains $N$ subcarrier frequencies with respect to the normalized carrier frequency $\tilde{f}_{m}, \odot$ denotes the element-wise dot product. In addition, we define

$$
\boldsymbol{q}_{m}(\tau)=\cos \left(2 \pi \boldsymbol{f}_{m} \tau\right), \quad \boldsymbol{p}_{m}(\tau)=\sin \left(2 \pi \boldsymbol{f}_{m} \tau\right)
$$

Then, letting the relative delay as $\Delta \tau=\tau_{2}-\tau_{1}$, the FIM based on a single band OFDM signal in a two-path channel be derived by

$$
\begin{aligned}
\boldsymbol{F}_{m}(\boldsymbol{\theta}) & =\frac{2}{\sigma^{2}}\left[\begin{array}{ll}
\boldsymbol{A}_{m} & \boldsymbol{B}_{m} \\
\boldsymbol{C}_{m} & \boldsymbol{D}_{m}
\end{array}\right] \\
{\left[\boldsymbol{F}_{m}(\boldsymbol{\theta})\right]_{i j} } & =\Re\left[\frac{\partial \boldsymbol{\mu}^{\mathrm{H}}(\boldsymbol{\theta})}{\partial \theta_{i}} \frac{\partial \boldsymbol{\mu}(\boldsymbol{\theta})}{\partial \theta_{j}}\right] .
\end{aligned}
$$

as the first term of (3) vanishes, and

$$
\begin{gathered}
\boldsymbol{A}_{m}=\alpha_{1}^{2} 4 \pi^{2}\left[\begin{array}{cc}
\boldsymbol{f}_{m}^{T} \boldsymbol{f}_{m} & \frac{\alpha_{2}}{\alpha_{1}} \boldsymbol{q}_{m}^{\mathrm{T}}(\Delta \tau) \boldsymbol{f}_{m}^{2} \\
\frac{\alpha_{2}}{\alpha_{1}} \boldsymbol{q}_{m}^{\mathrm{T}}(\Delta \tau) \boldsymbol{f}_{m}^{2} & \frac{\alpha_{2}^{2}}{\alpha_{1}^{2}} \boldsymbol{f}_{m}^{\mathrm{T}} \boldsymbol{f}_{m}
\end{array}\right], \\
\boldsymbol{B}_{m}=\alpha_{1} 2 \pi\left[\begin{array}{cc}
0 & \boldsymbol{p}_{m}^{\mathrm{T}}(\Delta \tau) \boldsymbol{f}_{m} \\
-\frac{\alpha_{2}}{\alpha_{1}} \boldsymbol{p}_{m}^{\mathrm{T}}(\Delta \tau) \boldsymbol{f}_{m} & 0
\end{array}\right]=\boldsymbol{C}_{m}^{\mathrm{T}}, \\
\boldsymbol{D}_{m}=\left[\begin{array}{cc}
N & \mathbf{1}_{N}^{\mathrm{T}} \boldsymbol{q}_{m}(\Delta \tau) \\
\mathbf{1}_{N}^{\mathrm{T}} \boldsymbol{q}_{m}(\Delta \tau) & N
\end{array}\right] .
\end{gathered}
$$

The FIM depends on the relative delay $\Delta \tau$, but not on the absolute delay $\tau_{1}$.

Now we consider using multiple signal bands for time delay estimation. At the receiver, we can receive the signals from different bands simultaneously and stack them for time delay estimation. Here for convenience we assume that each band contains $N$ subcarriers for ranging, thus, there will be $N$ samples acquired in $\boldsymbol{r}_{m}(\boldsymbol{\theta})$. The expectation of the received signals from $M$ different bands is now written by

$$
\mathbb{E}\{\underline{\boldsymbol{r}}\}=\mathbb{E}\left\{\left[\begin{array}{c}
\underline{\boldsymbol{r}}_{1}(\boldsymbol{\theta}) \\
\underline{\boldsymbol{r}}_{2}(\boldsymbol{\theta}) \\
\vdots \\
\underline{\boldsymbol{r}}_{M}(\boldsymbol{\theta})
\end{array}\right]\right\}=\boldsymbol{\mu}(\boldsymbol{\theta}) \in \mathbb{C}^{N M \times 1} .
$$

Here, the noise from different bands is assumed to be independent and statistically identical. Then, the FIM based on multiple signal bands is just the sum of the FIM of each single band, which is written by

$$
\boldsymbol{F}(\boldsymbol{\theta})=\sum_{m=1}^{M} \boldsymbol{F}_{m}(\boldsymbol{\theta})=\frac{2}{\sigma^{2}}\left[\begin{array}{ll}
\sum_{m} \boldsymbol{A}_{m} & \sum_{m} \boldsymbol{B}_{m} \\
\sum_{m} \boldsymbol{C}_{m} & \sum_{m}^{m} \boldsymbol{D}_{m}
\end{array}\right],
$$

in which $\boldsymbol{F}_{m}(\boldsymbol{\theta})$ denotes the FIM from the $m$-th band in a twopath channel (see (7)). Eventually, the CRLB of the estimators, with among them the unknown propagation delay of the LoS path, can be derived from (2).
The CRLB is a measure for the ranging accuracy and the capability of distinguishing the LoS path and the reflections, and will be used to design a sparse multiband signal in the following section.

\section{Signal Design BASED on Convex Optimization}

Considering spectrum efficiency, it may not be necessary to occupy an entire wide signal bandwidth for time delay estimation. Thus, similar to [6], we introduce a binary selection vector for $M$ different OFDM bands,

$$
\boldsymbol{w}=\left[\begin{array}{llll}
w_{1} & w_{2} & \ldots & w_{M}
\end{array}\right]^{\mathrm{T}} \in\{0,1\}^{M},
$$

where $w_{m}=1(0)$ indicates that the signal from the $m$-th OFDM signal band is activated (muted), and used (not used) for time delay estimation. Rather than (9), the FIM based on a multiband signal with a selection vector can be obtained by

$$
\boldsymbol{F}(\boldsymbol{w}, \boldsymbol{\theta})=\sum_{m=1}^{M} w_{m} \boldsymbol{F}_{m}(\boldsymbol{\theta}) .
$$

To design the optimal multiband signal for ranging, we use the CRLB as an inequality constraint in the optimization problem to meet the desired performance, and try to minimize the number of activated signal bands.

However, the propagation delay and the gain in $\boldsymbol{\theta}$ have different units. If the uncertainty in one of the estimators is numerically much larger than one of the others, the optimization will be dominated by the estimators with the larger uncertainty. Thus, we also introduce a user specified compensation weight vector $\gamma[7]$ to balance the estimators,

$$
\widehat{C R L B}(\boldsymbol{\theta})=\operatorname{diag}\{\boldsymbol{\gamma}\} C R L B(\boldsymbol{\theta}) \operatorname{diag}\{\boldsymbol{\gamma}\} .
$$

Then, the modified FIM (MFIM) is defined as

$$
\tilde{\boldsymbol{F}}(\boldsymbol{\theta})=\operatorname{diag}^{-1}\{\boldsymbol{\gamma}\} \boldsymbol{F}(\boldsymbol{\theta}) \operatorname{diag}^{-1}\{\boldsymbol{\gamma}\} .
$$

Furthermore, for the purpose of ranging and positioning, we only use the time delay estimates to retrieve the geometric information. Although the gain of each path is also important to help us distinguish whether the propagation channel contains a direct-LoS path or not, its required accuracy can be much lower than that of the time delay estimators. Thus, the different requirements for the time estimators and the gain estimators should be also taken into account when we design the compensation vector $\gamma$. In our case, $\gamma_{\tau} \gg \gamma_{\alpha}$.

After the compensation, all estimators are expected to have approximately the same precision. Because of the presence of multiple unknown parameters, the CRLB is no longer a scalar. In order to employ the CRLB in an objective function or constraint in an optimization problem, the minimum eigenvalue constraint (i.e., E-optimality) [6], [7] is chosen here. The errors of the estimators in $\epsilon_{\boldsymbol{\theta}}$ are assumed to be constrained in an origin-centered sphere of radius $r_{e}$, with a probability larger than $p_{e}$, which can be written by

$$
p\left(\left\|\boldsymbol{\epsilon}_{\boldsymbol{\theta}}\right\|_{2} \leq r_{e}\right) \geq p_{e} .
$$


Then the minimum eigenvalue for the MFIM is given in [7] and also derived in appendix B,

$$
\lambda_{\min }\{\tilde{\boldsymbol{F}}(\boldsymbol{\theta})\} \geq \lambda_{\text {eig }}=\frac{N_{u}}{r_{e}^{2}}\left(\frac{1}{1-p_{e}}\right),
$$

where $N_{u}$ denotes the number of unknown parameters in $\boldsymbol{\theta}$. Since here we are using the MFIM which is the inverse of the CRLB, the smallest eigenvalue of the MFIM should be larger than the $\lambda_{\text {eig }}$ derived in (15). Thus, considering $M$ available signal bands for time delay estimation, the inequality constraint can be written as

$$
\sum_{m=1}^{M} w_{m} \tilde{\boldsymbol{F}}_{m}(\boldsymbol{\theta})-\lambda_{\text {eig }} \mathcal{I}_{N_{u}} \succeq \mathbf{0}_{N_{u}} .
$$

Based on the actual positioning scenario or the user desired capability of separating certain reflections, a set of $\mathcal{U}$, containing different potential relative delays and corresponding gains, is introduced here to compute a numerical value for the MFIM in the optimization, using (9) and (13).

In order to design a sparse multiband signal for ranging, the objective function is based on the $l_{1}$ norm, which can produce a sparse selection vector. The optimization problem is now formulated by

$$
\begin{aligned}
\underset{\boldsymbol{g} \in \mathbb{R}^{M}}{\arg \min } & \|\boldsymbol{g}\|_{1} \\
\text { s.t. } & \sum_{m=1}^{M} g_{m} \tilde{\boldsymbol{F}}_{m}(\boldsymbol{\theta})-\lambda_{\text {eig }} \mathcal{I}_{N_{u}} \succeq \mathbf{0}_{N_{u}} \quad \forall \boldsymbol{\theta} \in \mathcal{U} \\
& 0 \leq g_{m} \leq 1, \quad m=1,2, \ldots, M
\end{aligned}
$$

The selection vector $\boldsymbol{w}$ defined in (10) with elements being either zero or one, is a non-convex function, which is thus relaxed to inequalities. The value of the elements in a selection vector $\boldsymbol{g}$ can vary from zero to one. Afterwards, we simply round the float value in $\boldsymbol{g}$ to the integer number (i.e., 0 or 1) to obtain $\boldsymbol{w}$,

$$
w_{m}=\operatorname{round}\left(g_{m}\right) .
$$

We are satisfied by an approximation to the solution found in (18).

\section{Time Delay Estimation}

According to the resulting selection vector $\boldsymbol{w}$, multiple signal bands, sparsely placed in the available signal spectrum, are transmitted for time delay estimation and ranging. The propagation delay can be estimated through the matched filter method, however, the impact of multipath is not taken into account and will result in a possibly large bias. In order to mitigate the error introduced by multipath, we estimate the CIR by accommodating multiple signal copies.

According to the received signal $\underline{\boldsymbol{r}}_{m}$ shown in (8), we first estimate the channel frequency response for each subcarrier based on the known data $c_{i}$. The channel frequency response for the $m$-th band is denoted by $\underline{\boldsymbol{h}}_{m}$.

Since we only use a few signal bands that are sparsely placed in the spectrum, we can not simply use the ordinary inverse Fourier transform to estimate the CIR. Thus, in order to estimate the channel profile (i.e., delay and gain) from the channel frequency response, we can create a dictionary of phasors which have a constant amplitude but different propagation delays. Based on the dictionary, we can estimate the channel profile and further obtain the propagation delay of the direct-LoS path.

For example, considering a search span vector $s$ given by

$$
\boldsymbol{s}=\left[\begin{array}{llll}
0 & \Delta s & \ldots & \left(N_{s}-1\right) \Delta s
\end{array}\right],
$$

where $\Delta s$ denotes the time resolution of the dictionary, and $N_{s}$ denotes the number of delays in the dictionary.

Based on the search span vector $s$, the dictionary $\boldsymbol{A}$ is formulated into a $M N$-by- $N_{s}$ matrix, the $l$-th column of the dictionary $\left(l=1, \ldots, N_{s}\right)$ is given by

$$
[\boldsymbol{A}]_{l}=\left[\begin{array}{c}
\exp \left(-j 2 \pi \boldsymbol{f}_{m=1}(l-1) \Delta s\right) \\
\exp \left(-j 2 \pi \boldsymbol{f}_{m=2}(l-1) \Delta s\right) \\
\vdots \\
\exp \left(-j 2 \pi \boldsymbol{f}_{m=M}(l-1) \Delta s\right)
\end{array}\right] .
$$

The unknown parameters now are the gains for all possible paths with respect to the delays in the search span vector $s$, which is defined by an $N_{s}$-vector $\boldsymbol{x}$.

Generally, the number of possible delays in $s$ (i.e., column span of $\boldsymbol{A}$ ) is much larger than the number of observations,

$$
N_{s} \gg M N \text {. }
$$

When the actual number of paths $N_{p}$ in a multipath channel is much smaller than $N_{s}$, the estimates in $\boldsymbol{x}$ should be sparse. Thus, an $l_{1}$ norm is used as a penalty for $\boldsymbol{x}$ to produce a sparse solution. Then, the problem becomes to solve the following optimization problem

$$
\underset{\boldsymbol{x}}{\arg \min }\|\underline{\boldsymbol{h}}-\boldsymbol{A} \boldsymbol{x}\|_{2}^{2}+\lambda\|\boldsymbol{x}\|_{1},
$$

where the user specified $\lambda$ determines the level of sparsity of the estimation result. If the direct LoS path always exists in a multipath channel, its propagation delay can be determined from the path that has the strongest propagation gain.

\section{EXPERIMENT RESULTS}

In this section, ranging signals are designed for different scenarios, and experiments are carried out to evaluate the ranging performance. Here, we assume that all available signal bands are coherently transmitted and received through a single RF front-end. The experiments are based on the platform we recently developed in [8], which is using Ettus X310 USRPs (Universal Software Radio Peripherals) with an effective bandwidth of $160 \mathrm{MHz}$, and a sampling frequency $f_{s}$ of 200 MSamples/s.

To illustrate the performance of the proposed signal design, we use the system setups shown in Fig. 2, in which the transmitter and the receiver are synchronized via an 1 PPS and a $10 \mathrm{MHz}$ reference signal from the clock distributor. The central carrier frequency is fixed at $3.5 \mathrm{GHz}$ in the experiments for this paper, but it can be chosen differently. To emulate a simple two-path channel, one cable (c.1 in Fig.2(a)) connecting 
the transmitter and the receiver is the LoS path, and a dead-end cable (c. 2 in Fig.2(a)) is connected to create a single reflection. Furthermore, the sparse ranging signals can be also transmitted and received via antennas as shown in Fig. 2(b), and propagate through an actual multipath channel over the air.

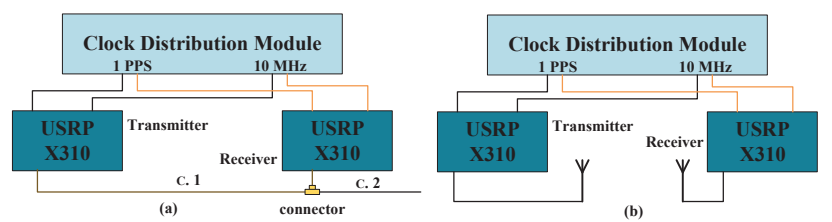

Fig. 2. Experiment setup (a) in a two path wired channel, which contains a direct cable connection between transmitter and receiver (c.1), and a dead-end cable (c.2) causing a reflection; (b) in a multipath radio channel, in which the signals are transmitted and received through the antennas.

We assume that an $160 \mathrm{MHz}$ signal bandwidth is available for ranging and positioning, offering 16 signal bands with a bandwidth of $10 \mathrm{MHz}$. Considering spectral efficiency, we wish to use as few signal bands as we can, but still achieve a favorable ranging accuracy.

\section{A. Two-path channel}

First, we consider a simple two-path channel, which contains a LoS path and one reflection. Based on the user specified requirements on the system performance, the procedure to formulate a convex optimization problem is presented in this subsection. Afterwards, the propagation delay is estimated based on the designed sparse ranging signal acquired from actual experiments.

The two-path channel is emulated by using the setup shown in Fig. 2(a). A $5 \mathrm{~m}$ cable c. 1 is used to produce a direct LoS path between a transmitter and a receiver. A $1 \mathrm{~m}$ cable c. 2 is connected to c. 1 by a connector for the reflection. Due to the bidirectional transmission in c.2, the reflected signal has propagated twice the cable length (i.e., $2 \mathrm{~m}$ ).

It should be mentioned that the propagation speed through the coaxial cable is different from the speed of light in vacuum. An oscilloscope with a $20 \mathrm{GHz}$ bandwidth is used to measure the propagation time across cable c.2 as a ground truth value. According to the measurement by the oscilloscope, the signal is expected to experience about 4 ns of delay within $1 \mathrm{~m}$ coaxial cable. Hence, due to the forward and backward propagation in c.2, the signal should experience about $8 \mathrm{~ns}$ of additional propagation delay.

Alternatively, instead of using the oscilloscope to determine the propagation delay, we can estimate the propagation delay based on the ranging signal. To design a sparse multiband signal, which is able to properly estimate the forward and backward propagation delay in a $1 \mathrm{~m}$ coaxial cable, we formulate a convex optimization as we proposed in section III.

As an example, for $70 \%$ of the cases (i.e., $P_{e}=0.7$ ), the error for the time delay estimator $\tau$ is required to be less than $0.04 \mathrm{~ns}$, and the error for the gain estimator $\alpha$ less than 0.05 .
Obviously, the delay estimator is numerically much smaller than the gain estimator. A compensation vector should be introduced to numerically adjust the required accuracy for the propagation delay and gain to the same order of magnitude. First, the unit of the propagation delay is scaled from second to sample interval, which is the inverse of the sampling frequency $f_{s}$. Then, to further balance the performance of delay and gain estimators, and maintain their error bounds on the same level, a scalar of 0.3 is introduced for the gain estimate. In such a condition, the compensation vector is determined as

$$
\gamma=\left[\begin{array}{llll}
f_{s} & f_{s} & 0.3 & 0.3
\end{array}\right]^{\mathrm{T}},
$$

where $f_{s}=200 \times 10^{6} \mathrm{~Hz}$ in the current experiment setup.

In addition, the ranging signal should be able to distinguish the LoS path and the reflection with a delay of about $8 \mathrm{~ns}$. To improve the robustness of the ranging signal, we expect the signal to be able to distinguish a reflection with a delay of from $7.9 \mathrm{~ns}$ to $8.1 \mathrm{~ns}$.

Here we assume that the signal attenuates $10 \mathrm{~dB}$ per $100 \mathrm{~m}$ in the coaxial cable, and the attenuation is proportional with the distance. Due to the forward and backward propagation in a $1 \mathrm{~m}$ coaxial cable (i.e., c.2), the reflection can experience an extra $-2 \mathrm{~dB}$ propagation gain compared with the LoS path (i.e., c.1). Thus, the linear relative gain is about 0.63 . Therefore, to compute the numerical value for the FIMs, the set of $\mathcal{U}$ in (17) contains the following sets,

$$
\boldsymbol{\theta}=\left[\begin{array}{llll}
0 & \tau_{2,1} & 1 & 0.63
\end{array}\right]^{\mathrm{T}}, \quad \tau_{2,1} \in[7.9,8.1] \mathrm{ns} .
$$

After we determine $\tilde{\boldsymbol{F}}(\boldsymbol{\theta})$ and $\lambda_{\text {eig }}$ in (17), the optimization problem can be solved by the CVX toolbox [9]. Fig. 3 shows the minimum signal bands we could use for ranging and can still achieve the required performance. Eventually, 4 bands of $10 \mathrm{MHz}$ each are used, which is able to distinguish a reflection with a relative delay of about $8 \mathrm{~ns}$, and still achieve a certain accuracy.

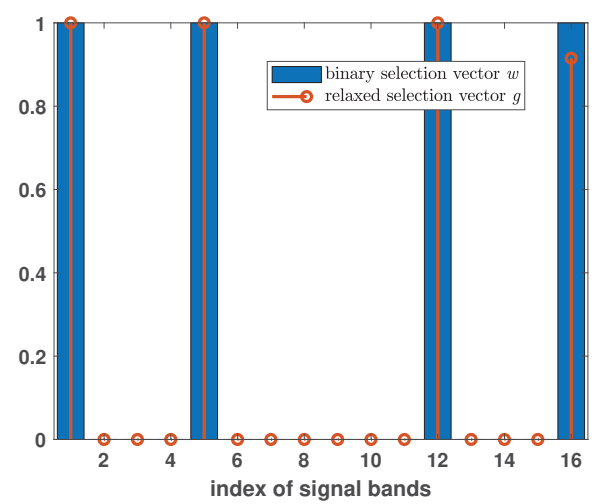

Fig. 3. The sparse signal bands derived from the convex optimization, considering a reflection with a delay of about $8 \mathrm{~ns}$.

The sparse multiband signal as shown in Fig. 3 is transmitted and received simultaneously using the setup presented in Fig. 2(a). Using the time delay estimation method presented 
in section IV, the magnitude of the channel impulse response is shown in Fig. 4 as a function of time in nano-second.

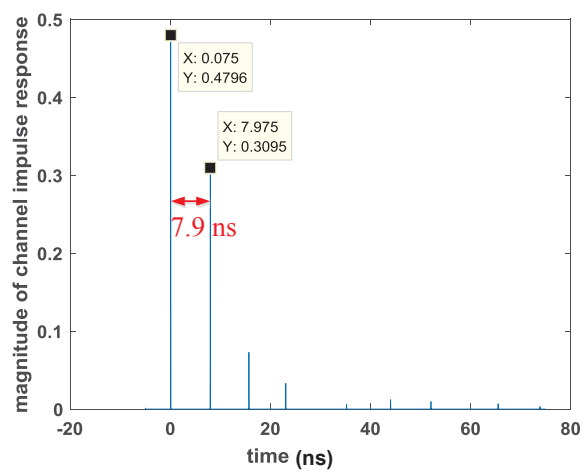

Fig. 4. Magnitude of channel impulse response, which is estimated from the sparse multiband signal shown in Fig.3. The relative delay forth and back in the $1 \mathrm{~m}$ coaxial cable is determined as $7.9 \mathrm{~ns}$.

To justify the ranging performance of the proposed sparse multiband signal in a two path channel, we only focus on the relative delay between the LoS path and the reflection, instead of the absolute propagation delay. After a time synchronization, an FFT window will be determined to obtain the channel frequency response. Thus, the delay of the LoS path shown in Fig. 4 is offset by a fraction of the sample interval. From this figure, we can also determine that the forward and backward propagation delay in c. 2 , which is equivalent to a 2 $\mathrm{m}$ coaxial cable, is $7.9 \mathrm{~ns}$, and close to the value measured by the oscilloscope.

It should be pointed out that the difference between the time delay measured by the oscilloscope and estimated using the proposed sparse ranging signal may be caused by different hardware connections, which introduce small but different delays. In fact, we may not simply use a dead-end cable c. 2 to emulate a perfect two-path channel; also the reflection of the reflection will be received, but with a much smaller propagation gain as shown in Fig. 4.

As a comparison, Fig. 5 (a) shows the estimated CIR based on only two edge signal bands in Fig. 3 (i.e., the 1-st band and the 16-th band), which has the same virtual signal bandwidth as the proposed sparse multiband signal. However, using the only two edge signal bands, the delay estimator for the LoS path is largely correlated with the one from the reflection, the system becomes unstable and sensitive to the noise. As shown in Fig. 5 (a), the reflection cannot be properly separated from the LoS path, and the relative delay between the LoS path and the reflection is estimated as $15.75 \mathrm{~ns}$. On the contrary, we also occupy all available 16 signal bands to estimate the channel impulse response of the given experiment setup. As shown in Fig. 5(b), the estimated relative delay is $7.875 \mathrm{~ns}$ and almost the same as the one computed based the designed sparse ranging signal.

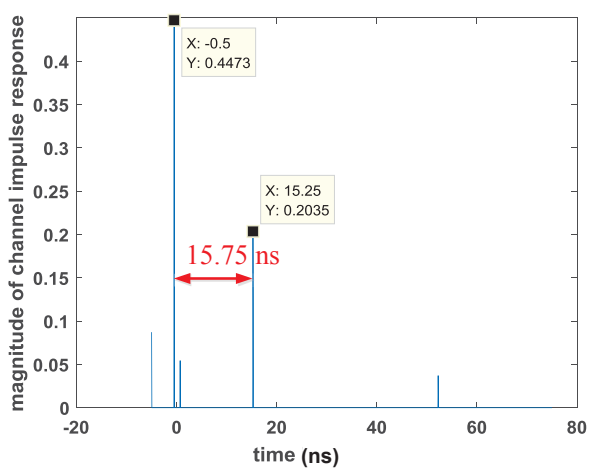

(a)

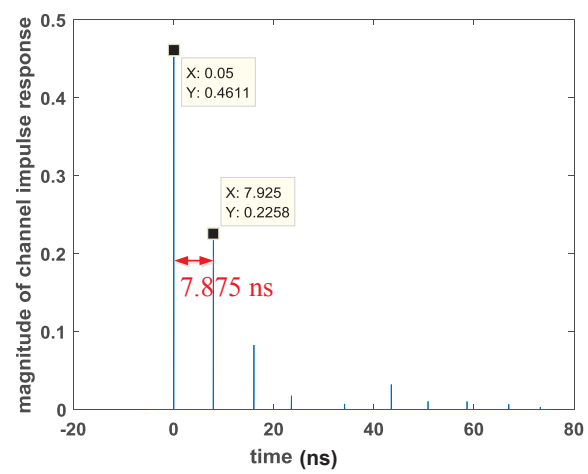

(b)

Fig. 5. Magnitude of the channel impulse response, (a) based on the two edge signal bands shown in Fig.3 (i.e., the 1-st band and the 16-th band). The forward and backward propagation delay in an $1 \mathrm{~m}$ coaxial cable is incorrectly determined as $15.75 \mathrm{~ns}$; (b) based of all 16 available signal bands. The relative delay is estimated to be $7.875 \mathrm{~ns}$.

\section{B. Multipath channel}

In the previous subsection, only a single reflection is considered in the ranging signal design. In practice, there are generally many reflections in an actual multipath channel. Similarly, to define the required performance, for $70 \%$ of the cases, the error of the delay estimator $\tau$ should be less than $0.33 \mathrm{~ns}$, which is equivalent to $10 \mathrm{~cm}$ in distance. The error of the gain estimator $\alpha$ is required to be less than 0.3 . Since the value of the time estimators is numerically much smaller than the one of the gain estimators, a similar compensation vector shown in (21) is applied here to adjust the accuracy of the propagation delay and gain to the same order of magnitude.

In a multipath channel, the ranging signal is expected to distinguish the reflections which are from $1 \mathrm{~m}$ to $10 \mathrm{~m}$ away from the LoS path. In addition, the gain of those reflections are required to compute numerical values for the FIMs in (17). The received signal power from different paths, for example with different geometric distance (i.e., $d_{1}$ and $d_{2}$ ), can be computed using a free-space path loss model [10],

$$
P_{r_{1}}=P_{t}\left[\frac{\lambda \sqrt{G_{l}}}{4 \pi d_{1}}\right]^{2}, \quad P_{r_{2}}=P_{t}\left[\frac{\lambda \sqrt{G_{l}}}{4 \pi d_{2}}\right]^{2},
$$


where $\lambda$ denotes the wavelength of the central carrier, $\sqrt{G_{l}}$ denotes the product of the antenna gain. Then, when $d_{1}$ is assumed to be the distance of the LoS path and $d_{2}$ the distance travelled by the reflection, the relative attenuation is derived as follows

$$
\alpha_{2,1}=\frac{\alpha_{2}}{\alpha_{1}}=\sqrt{\frac{P_{r_{2}}}{P_{r_{1}}}}=\frac{d_{1}}{d_{2}} .
$$

Here, the distance of the LoS path $d_{1}$ is assumed to be $0.3 \mathrm{~m}$, and the relative distance $d_{2}-d_{1}$ is expected to be from $1 \mathrm{~m}$ to $10 \mathrm{~m}$. Afterwards, the set of $\mathcal{U}$ in (17) should contain the following sets,

$$
\boldsymbol{\theta}=\left[\begin{array}{llll}
0 & \tau_{2,1} & 1 & \alpha_{2,1}
\end{array}\right]^{T}, \tau_{2,1}=\frac{d_{2}-d_{1}}{c}, \alpha_{2,1}=\frac{d_{1}}{d_{2}} .
$$

By solving the convex optimization formulated in (17), Fig. 6 shows the sparse multiband signal which are able to distinguish the reflection with a relative distance from $1 \mathrm{~m}$ to $10 \mathrm{~m}$.

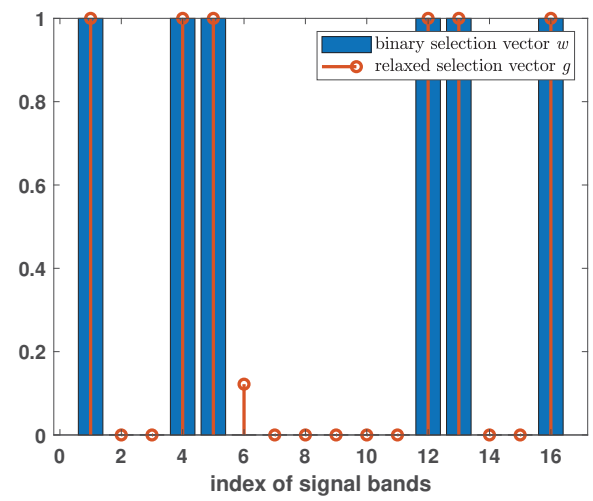

Fig. 6. The sparse multiband signal derived by the convex optimization considering a multipath channel.

To evaluate the ranging performance of the proposed sparse ranging signal shown in Fig. 6, the setup presented in Fig. 2(b) is used. Since the hardware delay has not yet been accurately calibrated, we only estimate the displacement of the receiver (i.e., track the change in distance) at different locations. Thus, the initial location of the receiver antenna is chosen as a reference point. In addition, a professional laser distometer is used to measure the ground truth value as shown in Fig. 7, which is the actual experiment setup in the Lab. The laser distometer, transmitter antenna and receiver antenna are placed on a straight line.

Similarly, the channel impulse responses at different locations are estimated based on the method presented in section IV. Fig. 8, as an example, shows the estimated channel impulse response in the lab. The most dominant relative delays between the LoS path and the reflections are generally less than $30 \mathrm{~ns}$, which is equivalent to about $9 \mathrm{~m}$ in distance, and is also in line with the user specified capability of distinguishing certain reflections.

Since it is assumed that the direct LoS path always presents in a multipath channel, the time of arrival is determined from

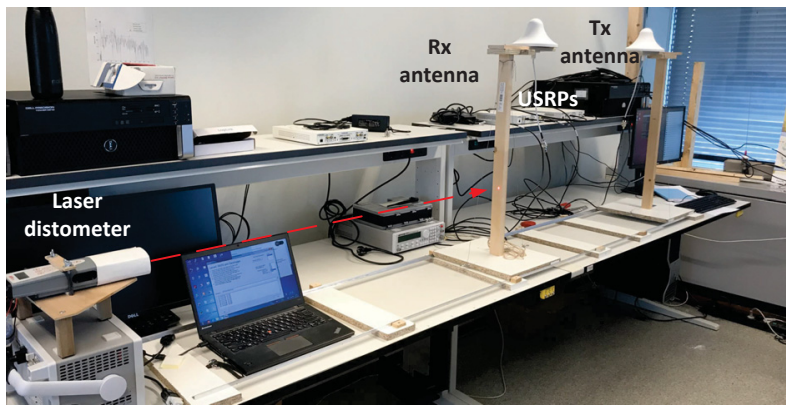

Fig. 7. Experimental setup in the lab. The ground truth values are measured by the laser distometer. The Rx antenna can be slide in a rail along a straight line.

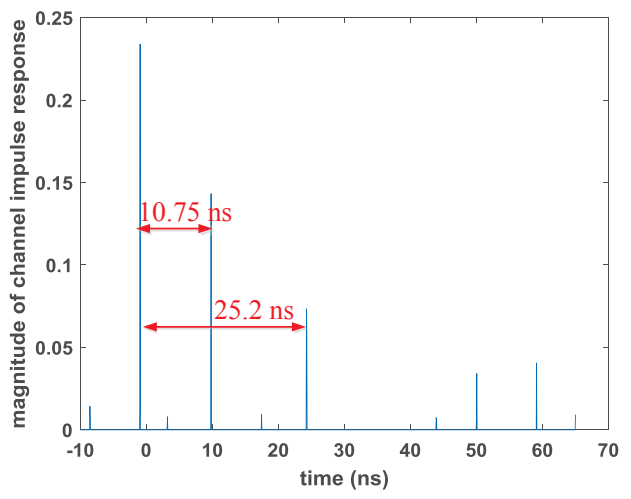

Fig. 8. Magnitude of the channel impulse response at one of the receiver locations shown in Fig. 7 in the lab.

the path that has the strongest propagation gain. By tracking the displacement of the receiver, Fig. 9 shows the ranging performance of using the designed sparse multiband signal. The ground-truth values are measured by the laser distometer, for which the typical measuring accuracy is specified to be a few millimeters. There are 42 receiver locations between the laser distometer and the transmit antenna shown in Fig. 7 that are recorded for the performance analysis. Comparing the propagation delay estimated from the sparse signal and the one retrieved from the laser distometer measurements, the root-mean-square error (RMSE) of the receiver displacement is $7.01 \mathrm{~cm}$, and satisfies the user specified ranging performance (i.e., $10 \mathrm{~cm}$ ).

\section{CONCLUSION}

In this paper, we proposed a sparse signal bands selection methodology for a time-based ranging and positioning system. The CRLB has been derived in a two-path channel and is used as a metric to formulate a convex optimization problem, which considers the correlation between the LoS path and the reflection. To improve the efficiency of spectrum usage and relax the requirement on computation power, we only select a few signal bands from the available signal spectrum for ranging, but still meet the desired accuracy and have the capability to distinguish certain reflections. The actual 


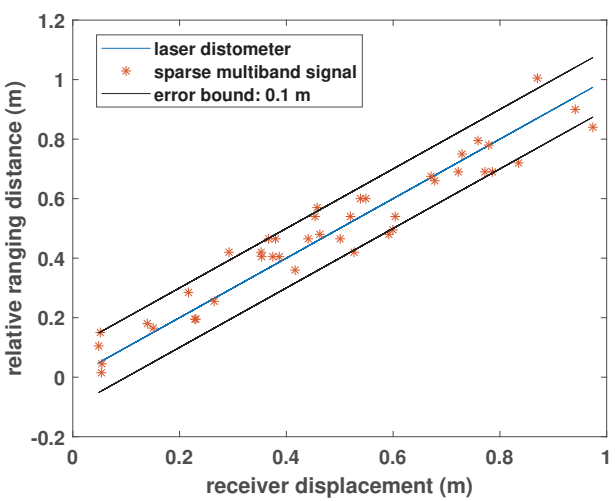

Fig. 9. The ranging performance of using the sparse multiband signal shown in Fig.6, where the horizontal axis stands for the displacements measured by the laser distometer and assumed to be ground truth values for this experiment.

experiments in a two path channel and a multipath channel are conducted to demonstrate the ranging performance of the proposed sparse ranging signals. A sub-decimeter ranging accuracy is achieved by using a sparse multiband signal with a virtual signal bandwidth of $160 \mathrm{MHz}$ with a spectrum use of $25 \%-40 \%$ in an indoor laboratory environment. It is expected that with larger virtual signal bandwidth, the relative spectrum use can be be reduced even further.

\section{APPENDIX A}

FIM OF SinglE BAND SIGNAL IN A TWO-PATH CHANNEL

In this appendix, we derive the elements of the FIM considering a multiband signal in a two-path channel. For the ease of derivation, the modulation on each subcarrier is assumed to be BPSK (i.e., $\left|c_{i}\right|^{2}=1$ ).

The first derivatives of mean $\boldsymbol{\mu}_{r}(\boldsymbol{\theta})$ with respect to the unknown parameters are given by

$$
\begin{aligned}
\frac{\partial \boldsymbol{\mu}_{m}(\boldsymbol{\theta})}{\partial \tau_{1}}= & -j \frac{2 \pi \alpha_{1}}{\sqrt{N}} \sum_{i=-N / 2}^{N / 2-1} c_{i} \exp \left(j 2 \pi \frac{i n}{N}\right)\left(\tilde{f}_{m}+f_{i}\right) \\
& \exp \left(-j 2 \pi\left(\tilde{f}_{m}+f_{i}\right) \tau_{1}\right) \\
\frac{\partial \boldsymbol{\mu}_{m}(\boldsymbol{\theta})}{\partial \tau_{2}}= & -j \frac{2 \pi \alpha_{2}}{\sqrt{N}} \sum_{i=-N / 2}^{N / 2-1} c_{i} \exp \left(j 2 \pi \frac{i n}{N}\right)\left(\tilde{f}_{m}+f_{i}\right) \\
& \exp \left(-j 2 \pi\left(\tilde{f}_{m}+f_{i}\right) \tau_{2}\right) \\
\frac{\partial \boldsymbol{\mu}_{m}(\boldsymbol{\theta})}{\partial \alpha_{1}}= & \frac{1}{\sqrt{N}} \sum_{i=-N / 2}^{N / 2-1} c_{i} \exp \left(j 2 \pi \frac{i n}{N}\right) \\
& \exp \left(-j 2 \pi\left(\tilde{f}_{m}+f_{i}\right) \tau_{1}\right) \\
\frac{\partial \boldsymbol{\mu}_{m}(\boldsymbol{\theta})}{\partial \alpha_{2}}= & \frac{1}{\sqrt{N}} \sum_{i=-N / 2}^{N / 2-1} c_{i} \exp \left(j 2 \pi \frac{i n}{N}\right) \\
& \exp \left(-j 2 \pi\left(\tilde{f}_{m}+f_{i}\right) \tau_{2}\right)
\end{aligned}
$$

where $\tilde{f}_{m}$ is the normalized central carrier frequency defined in (6).

Considering a single band OFDM signal in a two-path channel, the elements of the FIM can be derived as follows.

$$
\begin{aligned}
& \frac{\partial \boldsymbol{\mu}_{m}^{\mathrm{H}}(\boldsymbol{\theta})}{\partial \tau_{1}} \frac{\partial \boldsymbol{\mu}_{m}(\boldsymbol{\theta})}{\partial \tau_{1}}=\alpha_{1}^{2} 4 \pi^{2} \sum_{i=-N / 2}^{N / 2-1}\left(f_{i}+\tilde{f}_{m}\right)^{2} \\
& \frac{\partial \boldsymbol{\mu}_{m}^{\mathrm{H}}(\boldsymbol{\theta})}{\partial \tau_{1}} \frac{\partial \boldsymbol{\mu}_{m}(\boldsymbol{\theta})}{\partial \tau_{2}}=\alpha_{1} \alpha_{2} 4 \pi^{2} \sum_{i=-N / 2}^{N / 2-1}\left(f_{i}+\tilde{f}_{m}\right)^{2} \\
& \exp \left(-j 2 \pi\left(f_{i}+\tilde{f}_{m}\right)\left(\tau_{1}-\tau_{2}\right)\right) \\
& \frac{\partial \boldsymbol{\mu}_{m}^{\mathrm{H}}(\boldsymbol{\theta})}{\partial \tau_{1}} \frac{\partial \boldsymbol{\mu}_{m}(\boldsymbol{\theta})}{\partial \alpha_{1}}=-j \alpha_{1} 2 \pi \sum_{i=-N / 2}^{N / 2-1}\left(f_{i}+\tilde{f}_{m}\right) \\
& \frac{\partial \boldsymbol{\mu}_{m}^{\mathrm{H}}(\boldsymbol{\theta})}{\partial \tau_{1}} \frac{\partial \boldsymbol{\mu}_{m}(\boldsymbol{\theta})}{\partial \alpha_{2}}=-j \alpha_{1} 2 \pi \sum_{i=-N / 2}^{N / 2-1}\left(f_{i}+\tilde{f}_{m}\right) \\
& \exp \left(-j 2 \pi\left(f_{i}+\tilde{f}_{m}\right)\left(\tau_{1}-\tau_{2}\right)\right) \\
& \frac{\partial \boldsymbol{\mu}_{m}^{\mathrm{H}}(\boldsymbol{\theta})}{\partial \tau_{2}} \frac{\partial \boldsymbol{\mu}_{m}(\boldsymbol{\theta})}{\partial \tau_{2}}=\alpha_{2}^{2} 4 \pi^{2} \sum_{i=-N / 2}^{N / 2-1}\left(f_{i}+\tilde{f}_{m}\right)^{2} \\
& \frac{\partial \boldsymbol{\mu}_{m}^{\mathrm{H}}(\boldsymbol{\theta})}{\partial \tau_{2}} \frac{\partial \boldsymbol{\mu}_{m}(\boldsymbol{\theta})}{\partial \alpha_{1}}=j \alpha_{2} 2 \pi \sum_{i=-N / 2}^{N / 2-1}\left(f_{i}+\tilde{f}_{m}\right) \\
& \exp \left(-j 2 \pi\left(f_{i}+\tilde{f}_{m}\right)\left(\tau_{1}-\tau_{2}\right)\right) \\
& \frac{\partial \boldsymbol{\mu}_{m}^{\mathrm{H}}(\boldsymbol{\theta})}{\partial \tau_{2}} \frac{\partial \boldsymbol{\mu}_{m}(\boldsymbol{\theta})}{\partial \alpha_{2}}=-j \alpha_{2} 2 \pi \sum_{i=-N / 2}^{N / 2-1}\left(f_{i}+\tilde{f}_{m}\right) \\
& \frac{\partial \boldsymbol{\mu}_{m}^{\mathrm{H}}(\boldsymbol{\theta})}{\partial \alpha_{1}} \frac{\partial \boldsymbol{\mu}_{m}(\boldsymbol{\theta})}{\partial \alpha_{1}}=\frac{\partial \boldsymbol{\mu}_{m}^{\mathrm{H}}(\boldsymbol{\theta})}{\partial \alpha_{2}} \frac{\partial \boldsymbol{\mu}_{m}(\boldsymbol{\theta})}{\partial \alpha_{2}}=N \\
& \frac{\partial \boldsymbol{\mu}_{m}^{\mathrm{H}}(\boldsymbol{\theta})}{\partial \alpha_{1}} \frac{\partial \boldsymbol{\mu}_{m}(\boldsymbol{\theta})}{\partial \alpha_{2}}=\sum_{i=-N / 2}^{N / 2-1} \exp \left(-j 2 \pi\left(f_{i}+\tilde{f}_{m}\right)\left(\tau_{1}-\tau_{2}\right)\right)
\end{aligned}
$$

\section{APPENDIX B}

\section{Minimum Eigenvalue}

Considering the following random $n$ dimensional vector $\underline{x}$

$$
\underline{\boldsymbol{x}} \sim \mathcal{N}\left(\overline{\boldsymbol{x}} \in \mathbb{R}^{n}, \boldsymbol{Q}_{x x} \in \mathbb{R}^{n \times n}\right)
$$

we can have the following inequality (i.e., Chebyshev's inequality) [11]

$$
P\left(\|\underline{\boldsymbol{x}}-\overline{\boldsymbol{x}}\|_{2}^{2} \geq \epsilon^{2}\right) \leq \frac{\operatorname{trace}\left(\boldsymbol{Q}_{x x}\right)}{\epsilon^{2}},
$$

and

$$
P\left(\|\underline{\boldsymbol{x}}-\overline{\boldsymbol{x}}\|_{2}^{2} \leq \epsilon^{2}\right) \geq 1-\frac{\operatorname{trace}\left(\boldsymbol{Q}_{x x}\right)}{\epsilon^{2}} .
$$

Now, the estimators are assumed to be constrained in an origin-centered circle of radius $\epsilon$, with a probability larger than $P_{\epsilon}$. Hence, we have

$$
P_{\epsilon} \geq 1-\frac{\operatorname{trace}\left(\boldsymbol{Q}_{x x}\right)}{\epsilon^{2}},
$$


and

$$
\operatorname{trace}\left(\boldsymbol{Q}_{x x}\right) \leq\left(1-P_{\epsilon}\right) \epsilon^{2} .
$$

Here, if the variance matrix $\boldsymbol{Q}_{x x}$ is the CRLB, we can derive the following inequality

$$
\operatorname{trace}\left(\boldsymbol{Q}_{x x}\right) \leq \frac{n}{\lambda_{\min }\{\mathrm{FIM}\}} \leq\left(1-P_{e}\right) \epsilon^{2},
$$

where $\lambda_{\min }\{$ FIM $\}$ denotes the minimum eigenvalue of the FIM. Now, we can derive the lower bound for the minimum eigenvalue of the FIM, which is given by

$$
\lambda_{\min }\{\mathrm{FIM}\} \geq \frac{n}{\left(1-P_{e}\right) \epsilon^{2}} .
$$

\section{REFERENCES}

[1] M. S. Braasch, "Multipath," in Springer handbook of global navigation satellite systems, P. Teunissen and O. Montenbruck, Eds. Springer, 2017, ch. 15.

[2] F. Zanier, G. Bacci, and M. Luise, "Criteria to improve time-delay estimation of spread spectrum signals in satellite positioning," IEEE Journal of Selected Topics in Signal Processing, vol. 3, no. 5, pp. 748763, 2009

[3] H. Dun, C. Tiberius, G. Janssen, and C. Diouf, "Time delay estimation based on multi-band multi-carrier signal in multipath environments," in Proceedings of the 32nd International Technical Meeting of the Satellite Division of The Institute of Navigation (ION GNSS+ 2019), September 2019, pp. $2299-2313$

[4] J. del Peral-Rosado, J. A. Lopez-Salcedo, F. Zanier, and G. SecoGranados, "Position accuracy of joint time-delay and channel estimators in lte networks," IEEE Access, vol. 6, pp. 25 185-25 199, 2018.

[5] S. M. Kay, Fundamentals of statistical signal processing. Prentice Hall PTR, 1993.

[6] S. P. Chepuri and G. Leus, "Sparsity-promoting sensor selection for nonlinear measurement models," IEEE Transactions on Signal Processing, vol. 63, no. 3, pp. 684-698, 2014.

[7] E. Tohidi, M. Coutino, S. P. Chepuri, H. Behroozi, M. M. Nayebi, and G. Leus, "Sparse antenna and pulse placement for colocated MIMO radar," IEEE Transactions on Signal Processing, vol. 67, no. 3, pp. 579593, 2018.

[8] C. Diouf, G. Janssen, T. Kazaz, H. Dun, F. Chamanzadeh, and C. Tiberius, "A 400 Msps SDR platform for prototyping accurate wideband ranging techniques," in Proceedings of the 16th workshop on positioning, Navigation and Communications (WPNC), October 2019 (in press).

[9] M. Grant and S. Boyd, "CVX: Matlab software for disciplined convex programming, version 2.1," http://cvxr.com/cvx, Mar. 2014.

[10] A. Goldsmith, Wireless communications. Cambridge university press, 2005.

[11] P. Teunissen, D. Simons, and C. Tiberius, "Probability and observation theory." 2009. 